\title{
Improved McClelland and Koolen-Moulton bounds for distance energy of a graph
}

\author{
G. Sridhara ${ }^{1}$ (D), M.R. Rajesh Kanna ${ }^{2 *}$ (D) R. Pradeep Kumar ${ }^{3,4}$ (D) and D. Soner Nandappa ${ }^{4}$ (D)
}

"Correspondence:

mr.rajeshkanna@gmail.com

${ }^{2}$ Department of Mathematics, Sri.D.

Devaraja Urs Governement First

Grade College, Hunsur, India

Full list of author information is

available at the end of the article

\section{Introduction}

Let $G$ be a simple undirected graph with $n$ vertices and $m$ edges. The distance between two vertices $v_{i}$ and $v_{j}$ is denoted by $d_{i j}$ and is defined as the length of the shortest path from $v_{i}$ to $v_{j}$. The distance sum $W(G)=\sum_{i<j} d_{i j}$ is called the Wiener index of the graph $G$. For simplicity, we write the Wiener index as $W$. The distance matrix of the graph $G$ is defined as $A(G)=A_{D}(G)=\left[d_{i j}\right]$. Clearly, $A_{D}(G)$ is a symmetric matrix. Its eigenvalues are called $D$-eigenvalues and are ordered in the form $\mu_{1} \geq \mu_{2} \geq \cdots \geq \mu_{n}$. The largest eigenvalue $\mu_{1}$ is called the distance spectral radius of the graph $G$. We also write its absolute eigenvalues in decreasing order as $\rho_{1} \geq \rho_{2} \geq \cdots \geq \rho_{n}$. Given a graph $G$, the distance energy of $G$ is defined by $\mathcal{E}_{D}(G)=\sum_{i=1}^{n}\left|\mu_{i}\right|=\sum_{i=1}^{n} \rho_{i}$. For any vertex $v_{i}$ in the connected graph $G$, the eccentricity $e\left(v_{i}\right)$ is the distance between $v_{i}$ and a vertex that is farthest from $v_{i}$ in $G$. The minimum eccentricity among the vertices of $G$ is called the radius of graph $G$, and the maximum eccentricity among the vertices is called the diameter of the graph $G$, which are respectively denoted by $\operatorname{rad}(G)$ and $\operatorname{diam}(G)$.

The distance energy is analogous to the ordinary energy of a graph $G$, which is defined as $\mathcal{E}(G)=\sum_{i=1}^{n}\left|\lambda_{i}\right|$, where $\lambda_{1} \geq \lambda_{2} \geq \cdots \geq \lambda_{n}$ are ordinary eigenvalues of $G$ obtained from its adjacency matrix. The studies on the graph energy can be seen in papers [5, 6]. For a detailed survey on applications to the graph energy, see [2-4, 7]. For the ordinary energy, the best known bounds are the Koolen and Moulton upper bound $[9,10]$ and the McClelland lower bound [12].

(c) The Author(s) 2018. This article is distributed under the terms of the Creative Commons Attribution 4.0 International License (http://creativecommons.org/licenses/by/4.0/), which permits unrestricted use, distribution, and reproduction in any medium, provided you give appropriate credit to the original author(s) and the source, provide a link to the Creative Commons license, and indicate if changes were made. 
For a connected graph $G$, the Koolen and Moulton upper bound [13] for the distance energy in terms of $W, M$, and $n$ is

$$
\mathcal{E}_{D}(G) \leq\left(\frac{2 W}{n}\right)+\sqrt{(n-1)\left(2 M-\left(\frac{2 W}{n}\right)^{2}\right)} \text { for } 2 W \geq n,
$$

where $M=\sum_{i<j}^{n} d_{i j}^{2}$.

In this paper, we show that the upper bound (1.1) can be modified to a better bound for all classes of graphs with $n^{2} \geq 4 m$. Further results on upper bounds can also be seen in [11].

The McClelland bounds [13] for the distance energy of a graph, which is true for any connected graph $G$, is

$$
\sqrt{2 M+n(n-1)|\operatorname{det}(A)|^{\frac{2}{n}}} \leq \mathcal{E}_{D}(G) \leq \sqrt{2 M n}
$$

The lower bound obtained in this paper is better than that of McClelland. For more studies on the distance energy, we refer to $[1,8,14]$.

We use the following two lemmas, which follow from the properties of distance eigenvalues.

Lemma 1.1 Let $G$ be a graph with $n \geq 3$ vertices and $m$ edges. Let $\mu_{1} \geq \mu_{2} \geq \cdots \geq \mu_{n}$ be $D$-eigenvalues of $G$. Then

$$
\sum_{i=1}^{n} \mu_{i}=0 \text { and } \sum_{i=1}^{n} \mu_{i}^{2}=2 M
$$

Lemma 1.2 If $\mu_{1}(G)$ is the distance spectral radius of the graph $G$, then $\mu_{1}(G) \geq \frac{2 W}{n}$.

Note that $M=\sum_{i<j}^{n} d_{i j}^{2} \geq \sum_{i<j}^{n} d_{i j}=W$ and $\sqrt{M}=\sqrt{\sum_{i<j}^{n} d_{i j}^{2}} \leq \sum_{i<j}^{n} d_{i j}=W$.

\section{The main results}

Lemma 2.1 If $\mu_{n}(G)$ is the smallest distance eigenvalue of the graph $G$ and $\rho_{n}(G)$ is the smallest absolute distance eigenvalue, then

$$
\mu_{n}(G) \leq \sqrt{\frac{2 W}{n}} \quad \text { and } \quad \rho_{n}(G) \leq|\operatorname{det}(A)|^{\frac{1}{n}}
$$

Proof For any nonzero vector $X$,

$$
\mu_{n}(G) \leq \min _{X \neq 0}\left(\frac{X^{\prime} A X}{X^{\prime} X}\right) \leq \sqrt{\frac{J^{\prime} A J}{J^{\prime} J}}=\sqrt{\frac{2 W}{n}},
$$

where $J$ is the unit $n \times 1$ matrix $J=[1,1,1, \ldots, 1]^{\prime}$. Now consider $\rho_{1} \rho_{2} \rho_{3} \ldots \rho_{n}=|\operatorname{det}(A)|$. Then $\rho_{n} \rho_{n} \rho_{n} \ldots \rho_{n} \leq \rho_{1} \rho_{2} \rho_{3} \ldots \rho_{n} \leq|\operatorname{det}(A)| \Rightarrow \rho_{n}(G) \leq|\operatorname{det}(A)|^{\frac{1}{n}}$.

Lemma 2.2 Let $G$ be a graph with $n \geq 3$ vertices and $m$ edges. For the largest and smallest distance eigenvalues $\mu_{1}$ and $\mu_{n}$ of $G, \mu_{1}+\mu_{n} \leq 2 \sqrt{\frac{M(n-2)}{n}}$. 
Proof For $D$-eigenvalues $\mu_{1} \geq \mu_{2} \geq \cdots \geq \mu_{n}$ of $G$, it is well known that $\sum_{i=1}^{n} \mu_{i}=0$ and $\sum_{i=1}^{n} \mu_{i}^{2}=2 M$. Using the Cauchy-Schwarz inequality for $\left(\mu_{2}, \mu_{3}, \ldots, \mu_{n-1}\right)$ and $\underbrace{(1,1, \ldots, 1)}_{(n-2) \text { times }}(n \geq 3)$ we have

$$
\left(\sum_{i=2}^{n-1} \mu_{i}\right)^{2} \leq\left(\sum_{i=2}^{n-1} 1\right)\left(\sum_{i=2}^{n-1} \mu_{i}^{2}\right)
$$

that is, $\left(-\mu_{1}-\mu_{n}\right)^{2} \leq(n-2)\left(2 M-\mu_{1}^{2}-\mu_{n}^{2}\right)$.

$$
\begin{aligned}
\therefore(n-2) 2 M & \geq\left(\mu_{1}+\mu_{n}\right)^{2}+(n-2)\left(\mu_{1}^{2}+\mu_{n}^{2}\right) \\
& =\left(\mu_{1}+\mu_{n}\right)^{2}+(n-2)\left(\left(\mu_{1}+\mu_{n}\right)^{2}-2 \mu_{1} \mu_{n}\right) \\
& =\left(\mu_{1}+\mu_{n}\right)^{2}(n-1)-2(n-2) \mu_{1} \mu_{n} .
\end{aligned}
$$

However, $\left(\frac{\mu_{1}+\mu_{n}}{2}\right)^{2} \geq \mu_{1} \mu_{n}$, which implies that $-\mu_{1} \mu_{n} \geq-\left(\frac{\mu_{1}+\mu_{n}}{2}\right)^{2}$.

Thus $(n-2) 2 M \geq\left(\mu_{1}+\mu_{n}\right)^{2}(n-1)-2(n-2) \frac{\left(\mu_{1}+\mu_{n}\right)^{2}}{4}=\left(\mu_{1}+\mu_{n}\right)^{2} \frac{n}{2}$.

Hence $\mu_{1}+\mu_{n} \leq 2 \sqrt{\frac{M(n-2)}{n}}$.

\section{Upper bound for the distance energy of a graph}

Theorem 3.1 Let $G$ be a graph with $n \geq 3$ vertices and $m$ edges. If $n^{2} \geq 4 m$, then

$$
\mathcal{E}_{D}(G) \leq \frac{2 W}{n}+\sqrt{\frac{2 W}{n}}+\sqrt{(n-2)\left(2 M-\frac{2 W}{n}-\frac{4 W^{2}}{n^{2}}\right)} .
$$

The equality holds iff $G$ is $\frac{n}{2} K_{2}$.

Proof Applying the Cauchy-Schwarz inequality for $\left(\left|\mu_{2}\right|,\left|\mu_{3}\right|, \ldots,\left|\mu_{n-1}\right|\right)$ and $(\underbrace{(1,1, \ldots, 1)}_{(n-2) \text { times }}$, we have

$$
\begin{aligned}
& \left(\sum_{i=2}^{n-1}\left|\mu_{i}\right|\right)^{2} \leq\left(\sum_{i=2}^{n-1} 1\right)\left(\sum_{i=2}^{n-1}\left|\mu_{i}\right|^{2}\right), \\
& \left(\mathcal{E}_{D}(G)-\left|\mu_{1}\right|-\left|\mu_{n}\right|\right)^{2} \leq(n-2)\left(2 M-\left|\mu_{1}\right|^{2}-\left|\mu_{n}\right|^{2}\right), \\
& \mathcal{E}_{D}(G) \leq\left|\mu_{1}\right|+\left|\mu_{n}\right|+\sqrt{(n-2)\left(2 M-\left|\mu_{1}\right|^{2}-\left|\mu_{n}\right|^{2}\right)} .
\end{aligned}
$$

Let $\left|\mu_{1}\right|=x$ and $\left|\mu_{n}\right|=y$.

We maximize the function $f(x, y)=x+y+\sqrt{(n-2)\left(2 M-x^{2}-y^{2}\right)}$. Differentiating $f(x, y)$ with respect to $x$ and $y$, we have

$$
\begin{aligned}
& f_{x}=1-\frac{x(n-2)}{\sqrt{(n-2)\left(2 M-x^{2}-y^{2}\right)}}, \quad f_{y}=1-\frac{y(n-2)}{\sqrt{(n-2)\left(2 M-x^{2}-y^{2}\right)}}, \\
& f_{x x}=-\frac{\sqrt{(n-2)}\left(2 M-y^{2}\right)}{\left(2 M-x^{2}-y^{2}\right)^{\frac{3}{2}}}, \quad f_{y y}=-\frac{\sqrt{(n-2)}\left(2 M-x^{2}\right)}{\left(2 M-x^{2}-y^{2}\right)^{\frac{3}{2}}} \quad \text { and }
\end{aligned}
$$




$$
f_{x y}=-\frac{\sqrt{(n-2)}(x y)}{\left(2 M-x^{2}-y^{2}\right)^{\frac{3}{2}}} .
$$

For maxima or minima, $f_{x}=0$ and $f_{y}=0$, which implies

$$
x^{2}(n-1)+y^{2}=2 M \quad \text { and } \quad y^{2}(n-1)+x^{2}=2 M .
$$

Solving these equations, we obtain that $x=y=\sqrt{\frac{2 M}{n}}$. At this point the values of $f_{x x}, f_{y y}, f_{x y}$, and $\Delta=f_{x x} f_{y y}-\left(f_{x y}\right)^{2}$ are

$$
\begin{aligned}
& f_{x x}=-\frac{\sqrt{(n-2)}(n-1)}{\sqrt{\frac{2 M}{n}}(n-2)^{\frac{3}{2}}} \leq 0, \quad f_{y y}=-\frac{\sqrt{(n-2)}(n-1)}{\sqrt{\frac{2 M}{n}}(n-2)^{\frac{3}{2}}}, \\
& f_{x y}=-\frac{\sqrt{(n-2)}}{\sqrt{\frac{2 M}{n}}(n-2)^{\frac{3}{2}}} \quad \text { and } \quad \Delta=\frac{n\left(n^{2}+3-3 n\right)}{2 M(n-2)^{2}} \geq 0 .
\end{aligned}
$$

Therefore $f(x, y)$ attains its maximum value at $x=y=\sqrt{\frac{2 M}{n}}$, and this maximum value is $f\left(\sqrt{\frac{2 M}{n}}, \sqrt{\frac{2 M}{n}}\right)=\sqrt{2 M n}$.

However, $f(x, y)$ decreases in the intervals

$$
\sqrt{\frac{2 M}{n}} \leq x \leq \sqrt{M} \text { and } 0 \leq y \leq \sqrt{\frac{2 W}{n}} \leq \sqrt{\frac{2 M}{n}} \leq \sqrt{M} .
$$

Since $n^{2} \geq 4 m, m \leq W \leq M$, and $\sqrt{M} \leq W$, we have

$$
\sqrt{\frac{2 m}{n}} \leq \sqrt{\frac{2 M}{n}} \leq \frac{2 W}{n} \leq\left|\mu_{1}\right| \leq \sqrt{M}, \quad 0 \leq\left|\mu_{n}\right| \leq \sqrt{\frac{2 W}{n}} \leq \sqrt{\frac{2 M}{n}} \leq \sqrt{M} .
$$

Thus $f\left(\left|\mu_{1}\right|,\left|\mu_{n}\right|\right) \leq f\left(\frac{2 W}{n}, \sqrt{\frac{2 W}{n}}\right) \leq f\left(\sqrt{\frac{2 M}{n}}, \sqrt{\frac{2 M}{n}}\right)$

$$
\Rightarrow \quad \mathcal{E}_{D}(G) \leq \frac{2 W}{n}+\sqrt{\frac{2 W}{n}}+\sqrt{(n-2)\left(2 m-\frac{2 W}{n}-\frac{4 W^{2}}{n^{2}}\right)} \leq \sqrt{2 M n} .
$$

For the graph $G \simeq \frac{n}{2} K_{2}(n=2 m), \mathcal{E}_{D}(G)=n$. Hence the equality holds.

Now we show that the above bound is an improvement of the Koolen-Moulton bound. Take $g(x, y)=x+y+\sqrt{(n-1)\left(2 M-x^{2}-y^{2}\right)}$. Then, clearly, $f(x, y) \leq g(x, y)$ for all $(x, y)$ in the given region of $x$ and $y$.

Along $x=\frac{2 W}{n}, f\left(\frac{2 W}{n}, y\right)=\frac{2 W}{n}+y+\sqrt{(n-2)\left(2 M-\frac{4 W^{2}}{n^{2}}-y^{2}\right)}$. However, $f\left(\frac{2 W}{n}, y\right)$ decreases in the interval $0 \leq y \leq \sqrt{2 M-\frac{4 M^{2}}{n^{2}}}$. Since $n^{2} \geq 4 m$, we also have $0 \leq y \leq \sqrt{\frac{2 W}{n}} \leq$ $\sqrt{2 M-\frac{4 W^{2}}{n^{2}}}$. Thus $f\left(\frac{2 W}{n}, \sqrt{\frac{2 W}{n}}\right) \leq f\left(\frac{2 W}{n}, 0\right)$.

Since $f\left(\frac{2 W}{n}, 0\right) \leq g\left(\frac{2 W}{n}, 0\right)$ and $g\left(\frac{2 W}{n}, 0\right)=\frac{2 W}{n}+\sqrt{(n-1)\left(2 M-\frac{4 W^{2}}{n^{2}}\right)}$, it follows that $f\left(\frac{2 W}{n}, \sqrt{\frac{2 W}{n}}\right) \leq g\left(\frac{2 W}{n}, 0\right)$. Hence

$$
\frac{2 W}{n}+\sqrt{\frac{2 W}{n}}+\sqrt{(n-2)\left(2 M-\frac{2 m}{n}-\frac{4 W^{2}}{n^{2}}\right)} \leq\left(\frac{2 W}{n}\right)+\sqrt{(n-1)\left(2 M-\left(\frac{2 W}{n}\right)^{2}\right)} .
$$




\section{Lower bounds for the distance energy of a graph}

Theorem 4.1 If $G$ is a nonsingular graph, then $\mathcal{E}_{D}(G) \geq n|\operatorname{det} A|^{\frac{1}{n}}$. The equality holds iff $G$ is $\frac{n}{2} K_{2}$, where $n=2 m$.

Proof For the eigenvalues $\rho_{1} \geq \rho_{2} \geq \cdots \geq \rho_{n}$ of $G$ (or its adjacency distance matrix $A$ ) it is well known that $|\operatorname{det}(A)|=\rho_{1} \rho_{2} \ldots \rho_{n}$. Since $G$ is nonsingular, we have $|\operatorname{det}(A)| \neq 0$.

Applying the Cauchy-Schwarz inequality for $n$ terms $a_{i}=\sqrt{\rho_{i}}$ and $b_{i}=1$ for all $i=$ $1,2, \ldots, n$, We have

$$
\begin{aligned}
& \sum_{i=1}^{n} \sqrt{\rho_{i}} \leq \sqrt{\left(\sum_{i=1}^{n} \rho_{i}\right) n}, \\
& \sqrt{\mathcal{E}_{D}(G)} \geq \frac{\sum_{i=1}^{n} \sqrt{\rho_{i}}}{\sqrt{n}} .
\end{aligned}
$$

However, $\frac{\sqrt{\rho_{1}}+\sqrt{\rho_{2}}+\cdots+\sqrt{\rho_{n}}}{n} \geq\left(\sqrt{\rho_{1} \rho_{2} \ldots \rho_{n}}\right)^{\frac{1}{n}}$,

$$
\sqrt{\mathcal{E}_{D}(G)} \geq \frac{n\left(\sqrt{\rho_{1} \rho_{2} \ldots \rho_{n}}\right)^{\frac{1}{n}}}{\sqrt{n}} \Rightarrow \mathcal{E}_{D}(G) \geq n|\operatorname{det} A|^{\frac{1}{n}}
$$

For the graph $G \simeq \frac{n}{2} K_{2}$ with $n=2 m,|\operatorname{det}(A)|=1$. Hence the equality holds.

Theorem 4.2 Let $G$ be a graph with $n>1$ vertices and $m$ edges, and let $2 W \geq n$. Then

$$
\mathcal{E}_{D}(G) \geq \frac{2 W}{n}+\frac{(n-1)|\operatorname{det}(A)|^{\frac{1}{(n-1)}}}{\left(\frac{2 W}{n}\right)^{\frac{1}{(n-1)}}}
$$

The equality holds if $G$ is isomorphic to $K_{n}$ and $\frac{n}{2} K_{2}$ with $n=2 m$.

Proof Using the Cauchy-Schwarz inequality for $\sqrt{\rho_{2}}, \sqrt{\rho_{3}}, \ldots, \sqrt{\rho_{n}}$ and $\underbrace{(1,1, \ldots, 1)}_{(n-1) \text { times }}$, we have

$$
\begin{aligned}
& \sum_{i=2}^{n} \sqrt{\rho_{i}} \leq \sqrt{\left(\sum_{i=2}^{n} \rho_{i}\right)(n-1),} \\
& \sum_{i=2}^{n} \sqrt{\rho_{i}} \leq \sqrt{\left(\mathcal{E}_{D}(G)-\rho_{1}\right)(n-1)}, \\
& \sqrt{\mathcal{E}_{D}(G)-\rho_{1}} \geq \frac{\sum_{i=2}^{n} \sqrt{\rho_{i}}}{\sqrt{n-1}} .
\end{aligned}
$$

However, $\frac{\sqrt{\rho_{2}}+\sqrt{\rho_{3}}+\cdots+\sqrt{\rho_{n}}}{n-1} \geq\left(\sqrt{\rho_{2} \rho_{3} \ldots \rho_{n}}\right)^{\frac{1}{n-1}}$, and therefore

$$
\begin{aligned}
& \sqrt{\mathcal{E}_{D}(G)-\rho_{1}} \geq \frac{(n-1)\left(\sqrt{\rho_{2} \rho_{3} \ldots \rho_{n}}\right)^{\frac{1}{(n-1)}}}{\sqrt{(n-1)}}, \\
& \mathcal{E}_{D}(G) \geq \rho_{1}+(n-1)\left(\rho_{2} \rho_{3} \ldots \rho_{n}\right)^{\frac{1}{(n-1)}}=\rho_{1}+(n-1)\left(\frac{|\operatorname{det}(A)|}{\rho_{1}}\right)^{\frac{1}{(n-1)}} .
\end{aligned}
$$


Let $\rho_{1}=x$ and $f(x)=x+(n-1)\left(\frac{|\operatorname{det}(A)|}{x}\right)^{\frac{1}{(n-1)}}$. Then $f^{\prime}(x)=1-\frac{|\operatorname{det}(A)|^{\frac{1}{(n-1)}}}{x^{\frac{n}{(n-1)}}}$ and $f^{\prime \prime}(x)=$ $\frac{n|\operatorname{det}(A)|^{\frac{1}{(n-1)}}}{(n-1) x^{\frac{(2 n-1)}{(n-1)}}}$.

For maxima or minima, $f^{\prime}(x)=0$, which gives the value $x=|\operatorname{det}(A)|^{\frac{1}{n}}$.

At this point, $f^{\prime \prime}(x)=\frac{n}{(n-1)}|\operatorname{det}(A)|^{\frac{-1}{n}} \geq 0$ for all $n>1$. Thus the function $f(x)$ attains its minimum at $x=|\operatorname{det}(A)|^{\frac{1}{n}}$, and the minimum value is $f\left(|\operatorname{det}(A)|^{\frac{1}{n}}\right)=n|\operatorname{det}(A)|^{\frac{1}{n}}$. However, $\frac{2 M}{n}=\frac{\rho_{1}^{2}+\rho_{2}^{2}+\cdots+\rho_{n}^{2}}{n} \geq \frac{2 W}{n} \geq \frac{\rho_{1}+\rho_{2}+\cdots+\rho_{n}}{n} \geq\left(\rho_{1} \rho_{2} \ldots \rho_{n}\right)^{\frac{1}{n}}$. This implies $|\operatorname{det}(A)|^{\frac{1}{n}} \leq \frac{2 W}{n}$. Since $2 W \geq n$, we have $\frac{2 W}{n} \leq \rho_{1}$.

Therefore, the function is increasing in the interval $|\operatorname{det} A|^{\frac{1}{n}} \leq \frac{2 W}{n} \leq \rho_{1} \leq \sqrt{2 M}$, and therefore $f\left(\rho_{1}\right) \geq f\left(\frac{2 W}{n}\right)$, and

$$
\mathcal{E}_{D}(G) \geq \frac{2 W}{n}+\frac{(n-1)|\operatorname{det}(A)|^{\frac{1}{(n-1)}}}{\left(\frac{2 W}{n}\right)^{\frac{1}{(n-1)}}}
$$

(i) If $G$ is isomorphic to $K_{n}$, then $|\operatorname{det}(A)|=n-1, \frac{2 W}{n}=n-1$, and hence $\mathcal{E}_{D}(G)=2(n-1)$.

(ii) If $G$ isomorphic to $\frac{n}{2} K_{2}$ with $n=2 m$, then the eigenvalues are \pm 1 (each with multiplicity $\left.\frac{n}{2}\right)$, and hence $\mathcal{E}_{D}(G)=n$.

Theorem 4.3 Let $G$ be a graph with $m$ edges and $n(>3)$ vertices, and let $W \geq n$. Then

$$
\mathcal{E}_{D}(G) \geq\left(\frac{W}{n}\right)+\left(\frac{2 W}{n}\right)+\frac{(n-2)|\operatorname{det}(A)|^{\frac{1}{(n-2)}}}{\left(\left(\frac{W}{n}\right)\left(\frac{2 W}{n}\right)\right)^{\frac{1}{(n-2)}}}
$$

Proof For $(n-2)$ entries of eigenvalues $\sqrt{\rho_{2}}, \sqrt{\rho_{3}}, \ldots, \sqrt{\rho_{n-1}}$ and $(1,1, \ldots, 1)$, applying the Cauchy-Schwarz inequality, we have $\underbrace{1,1,1)}_{(n-2) \text { times }}$

$$
\sum_{i=2}^{n-1} \sqrt{\rho_{i}} \leq \sqrt{\left(\sum_{i=2}^{n-1} \rho_{i}\right)(n-2)}
$$

that is,

$$
\sum_{i=2}^{n-1} \sqrt{\rho_{i}} \leq \sqrt{\left(\mathcal{E}_{D}(G)-\rho_{1}-\rho_{n}\right)(n-2)}
$$

so that

$$
\sqrt{\mathcal{E}_{D}(G)-\rho_{1}-\rho_{n}} \geq \frac{\sum_{i=2}^{n-1} \sqrt{\rho_{i}}}{\sqrt{n-2}}
$$

Since the arithmetic mean is greater than or equal to the geometric mean, we get

$$
\begin{aligned}
& \sqrt{\mathcal{E}_{D}(G)-\rho_{1}-\rho_{n}} \geq \frac{(n-2)\left(\sqrt{\rho_{2} \rho_{3} \cdots \rho_{n-1}}\right)^{\frac{1}{(n-2)}}}{\sqrt{(n-2)}}, \\
& \mathcal{E}_{D}(G) \geq \rho_{1}+\rho_{n}+(n-2)\left(\frac{|\operatorname{det}(A)|}{\rho_{1} \rho_{n}}\right)^{\frac{1}{(n-2)}} .
\end{aligned}
$$


The equality holds if $G$ is $\frac{n}{2} K_{2}$ with $n=2 m$ or $K_{n, n}$.

Put $\rho_{1}=x$ and $\rho_{n}=y$. We minimize the right side of the above function. Let $f(x, y)=$ $x+y+(n-2)\left(\frac{|\operatorname{det}(A)|}{x y}\right)^{\frac{1}{(n-2)}}$. Then $f_{x}=1-|\operatorname{det}(A)|^{\frac{1}{n-2}} y(x y)^{\frac{-(n-1)}{n-2}}$,

$$
\begin{aligned}
& f_{y}=1-|\operatorname{det}(A)|^{\frac{1}{n-2}} x(x y)^{\frac{-(n-1)}{n-2}}, \\
& f_{x x}=|\operatorname{det}(A)|^{\frac{1}{n-2}}\left(\frac{n-1}{n-2}\right) y^{2}(x y)^{\frac{-(2 n-3)}{n-2}}, \\
& f_{y y}=|\operatorname{det}(A)|^{\frac{1}{n-2}}\left(\frac{n-1}{n-2}\right) x^{2}(x y)^{\frac{-(2 n-3)}{n-2}} \text { and } \\
& f_{x y}=|\operatorname{det}(A)|^{\frac{1}{n-2}} \frac{(x y)^{\frac{-(n-1)}{n-2}}}{n-2} .
\end{aligned}
$$

For maxima or minima, $f_{x}=0$ and $f_{y}=0$, which gives $x y^{\frac{1}{n-1}}=|\operatorname{det}(A)|^{\frac{1}{n-1}}$ and $y x^{\frac{1}{n-1}}=$ $|\operatorname{det}(A)|^{\frac{1}{n-1}}$. Solving, we get $x=|\operatorname{det}(A)|^{\frac{1}{n}}$ and $y=|\operatorname{det}(A)|^{\frac{1}{n}}$. At this point, the values of $f_{x x}, f_{y y}, f_{x y}$, and $\Delta=f_{x x} f_{y y}-\left(f_{x y}\right)^{2}$ are $f_{x x}=f_{y y}=\left(\frac{n-1}{n-2}\right)|\operatorname{det}(A)|^{\frac{-1}{n}}, f_{x y}=\frac{1}{n-2}|\operatorname{det}(A)|^{\frac{-1}{n}}$, and $\Delta=\left(\frac{n}{n-2}\right)|\operatorname{det}(A)|^{\frac{-2}{n}} \geq 0$ for all $n \neq 2$. The minimum value is $f\left(|\operatorname{det}(A)|^{\frac{1}{n}},|\operatorname{det}(A)|^{\frac{1}{n}}\right)=$ $n|\operatorname{det}(A)|^{\frac{1}{n}}$. However, $|\operatorname{det}(A)|^{\frac{1}{n}} \leq \frac{2 W}{n} \leq \rho_{1} \leq \sqrt{2 M}$ and $0 \leq \rho_{n} \leq|\operatorname{det}(A)|^{\frac{1}{n}} \leq \frac{2 W}{n} \leq \sqrt{2 M}$.

For $W \geq n, f(x, y)$ increases in the intervals $|\operatorname{det}(A)|^{\frac{1}{n}} \leq \frac{2 W}{n} \leq x \leq \sqrt{2 M}$ and $0 \leq y \leq$ $|\operatorname{det}(A)|^{\frac{1}{n}} \leq \frac{W}{n} \leq \frac{2 W}{n} \leq \sqrt{2 M}$, that is, $f(x, y)$ increases in the intervals $|\operatorname{det}(A)|^{\frac{1}{n}} \leq \frac{2 W}{n} \leq$ $\rho_{1} \leq \sqrt{2 M}$ and $0 \leq \rho_{n} \leq|\operatorname{det}(A)|^{\frac{1}{n}} \leq \frac{W}{n} \leq \sqrt{2 M}$. At $\rho_{n}=\frac{W}{n}$, we have

$$
f\left(\rho_{1}, \rho_{n}\right) \geq f\left(\frac{2 W}{n}, \frac{W}{n}\right) \geq f\left(\frac{2 W}{n},|\operatorname{det}(A)|^{\frac{1}{n}}\right) \geq f\left(|\operatorname{det}(A)|^{\frac{1}{n}},|\operatorname{det}(A)|^{\frac{1}{n}}\right) .
$$

Therefore $\mathcal{E}_{D}(G) \geq\left(\frac{W}{n}\right)+\left(\frac{2 W}{n}\right)+\frac{(n-2) \mid \operatorname{det}(A))}{\left(\left(\frac{W}{n}\right)\left(\frac{1}{n}\right)\right)^{\frac{1}{(n-2)}}}$.

\section{Brief summary and conclusion}

In this paper, we established lower and upper bounds for the distance energy of a graph. Across the globe, attempts are being made by researchers to improve these bounds. The lower and upper bounds obtained in this paper improve the McClelland and KoolenMoulton bounds for the distance energy of a graph.

Acknowledgements

We thank anonymous reviewers and editors of this manuscript for giving their inputs and suggestions in improving the quality of this paper.

Funding

Not applicable.

\section{Competing interests}

The authors declare that they have no competing interests.

\section{Authors' contributions}

GS and MRR drafted the manuscript. RPK and DSN revised it. All authors read and approved the final manuscript.

\section{Author details}

${ }^{1}$ Post Graduate Department of Mathematics, Maharani's Science College for Women, Mysore, India. ${ }^{2}$ Department of Mathematics, Sri.D. Devaraja Urs Governement First Grade College, Hunsur, India. ${ }^{3}$ Department of Mathematics, Malnad College of Engineering, Hassan, India. ${ }^{4}$ Department of Mathematics, University of Mysore, Mysuru, India. 


\section{Publisher's Note}

Springer Nature remains neutral with regard to jurisdictional claims in published maps and institutional affiliations.

Received: 27 June 2018 Accepted: 30 November 2018 Published online: 06 December 2018

\section{References}

1. Bozkurt, S.B., Gügör, A.D., Zhou, B.: Note on distance energy of graph. MATCH Commun. Math. Comput. Chem. 64 129-134 (2010)

2. Cvetković, D., Gutman, I. (eds.): Applications of Graph Spectra. Mathematical Institution, Belgrade (2009)

3. Cvetković, D., Gutman, I. (eds.): Selected Topics on Applications of Graph Spectra. Mathematical Institute, Belgrade (2011)

4. Graovac, A., Gutman, I., Trinajstić, N.: Topological Approach to the Chemistry of Conjugated Molecules, vol. 4. Springer, Berlin (1977)

5. Gutman, I.: The energy of a graph. Ber. Math. Stat. Sekt. Forschungsz. Graz. 103, 1-22 (1978)

6. Gutman, I.: The energy of a graph: old and new. In: Betten, A., Kohnert, A., Laue, R., Wassermann, A. (eds.) Algebraic Combinatorics and Applications, pp. 196-211. Springer, Berlin (2001)

7. Gutman, I., Polansky, O.E.: Mathematical Concepts in Organic Chemistry. Springer, Berlin (1986)

8. Indulal, G., Gutman, I., Vijayakumar, A.: On the distance energy of graph. MATCH Commun. Math. Comput. Chem. 60, 461-472 (2008)

9. Koolen, J.H., Moulton, V.: Maximal energy of graphs. Adv. Appl. Math. 26, 47-52 (2001)

10. Koolen, J.H., Moulton, V.: Maximal energy of bipartite graphs. Graphs Comb. 19, 131-135 (2003)

11. Liu, H., Lu, M., Tian, F.: Some upper bounds for the energy of graphs. J. Math. Chem. 41(1), 45-57 (2007)

12. McClelland, B.J.: Properties of the latent root of a matrix: the estimation of $\pi$-electron energies. J. Chem. Phys. 54, 640-643 (1971)

13. Ramane, H.S., Revankar, D.S., Gutman, I., Rao, S.B., Acharya, B.D., Walikar, H.B.: Bounds for distance energy of graph. Kragujev. J. Math. 31, 59-68 (2008)

14. Zhou, B., llić, A.: On distance spectral radius and distance energy of graphs. MATCH Commun. Math. Comput. Chem. 64, 261-280 (2010)

\section{Submit your manuscript to a SpringerOpen ${ }^{0}$ journal and benefit from:}

- Convenient online submission

Rigorous peer review

- Open access: articles freely available online

- High visibility within the field

- Retaining the copyright to your article 